\title{
Two Japanese CADASIL Families Exhibiting Notch3 Mutation R75P Not Involving Cysteine Residue
}

\author{
Toshiki Mizuno ${ }^{1}$, Manabu Muranishi ${ }^{1}$, Torusunjian Torugun ${ }^{1}$, Hiromi Tango ${ }^{1}$, \\ Yoshinari Nagakane ${ }^{1}$, Tukasa Kudeken ${ }^{3}$, Yuji Kawase ${ }^{3}$, Kiyokazu Kawabe ${ }^{3}$, \\ Fumiko Oshima ${ }^{4}$, Takeshi Yaoi ${ }^{2}$, Kyoko Itoh ${ }^{2}$, \\ Shinji Fushiki ${ }^{2}$ and Masanori Nakagawa ${ }^{1}$
}

\begin{abstract}
Most previously reported mutations in cerebral autosomal dominant arteriopathy with subcortical infarcts and leukoencephalopathy (CADASIL) result in an odd number of cysteine residues within the epidermal growth factor (EGF)-like repeats in Notch3. We report here R75P mutation in two Japanese CADASIL families not directly involving cysteine residues located within the first EGF-like repeats. Probands in both families had repeated episodes of stroke, depression, dementia as well as T2 high-intensity lesions in the basal ganglia and periventricular white matter, but fewer white matter lesions in the temporal pole on MRI. These families provide new insights into the diagnosis and pathomechanisms of CADASIL.
\end{abstract}

Key words: CADASIL, Notch3, EGF-like repeat, cysteine, white matter lesion, GOM

(Inter Med 47: 2067-2072, 2008)

(DOI: 10.2169/internalmedicine.47.1391)

\section{Introduction}

Cerebral autosomal dominant arteriopathy with subcortical infarcts and leukoencephalopathy (CADASIL) is a familial adult-onset progressive vascular disorder characterized by recurrent ischemic attacks leading to vascular dementia (1). Joutel et al identified the defective gene in CADASIL as Notch3, which consists of 33 exons encoding a protein of 2321 amino acids, including 34 epidermal growth factor (EGF)-like repeats in the extracellular amino-terminal region (2). CADASIL is caused by single missense mutations (2-5), small in-frame deletions $(6,7)$, or splice site mutations (8) in the Notch3 gene. Most previously reported mutations have resulted in an odd number of cysteine residues within the first five EGF-like repeats $(4,9)$. Each missense mutation is predicted to replace the wild-type amino acid with a cysteine residue or to replace one of the six highly conserved cysteine residues with another amino acid in the
EGF-like repeats. These mutations are thought to change the conformation of EGF-like repeats due to altered covalent bonding between cysteine residues (9).

Brain magnetic resonance imaging (MRI) shows diffuse white matter abnormalities and small subcortical cystic lesions suggestive of infarcts in CADASIL patients (10-14). These lesions are predominantly located in the centrum semiovale, thalamus, basal ganglia and pons. Several reports have focused on temporopolar white matter lesions as a diagnosis marker for CADASIL, which allows the condition to be distinguished from other cerebrovascular diseases, such as Binswanger disease (12-15). Therefore, a clinician may suspect CADASIL in hereditary young stroke patients with these specific MRI findings. Here, we report two interesting Japanese CADASIL families exhibiting Notch3 mutation $\mathrm{R} 75 \mathrm{P}$ that does not directly involve cysteine residues, and fewer white matter lesions in the temporal pole on MRI.

\footnotetext{
${ }^{1}$ Department of Molecular Neurology, Kyoto Prefectural University of Medicine, Kyoto, ${ }^{2}$ Department of Pathology \& Applied Neurobiology, Kyoto Prefectural University of Medicine, Kyoto, ${ }^{3}$ Department of Neurology, Oomori Hospital, Toho University, Tokyo and ${ }^{4}$ Department of Neurology, Kyoto First Red Cross Hospital, Kyoto

Received for publication June 6, 2008; Accepted for publication August 18, 2008

Correspondence to Dr. Toshiki Mizuno, mizuno@koto.kpu-m.ac.jp
} 
A

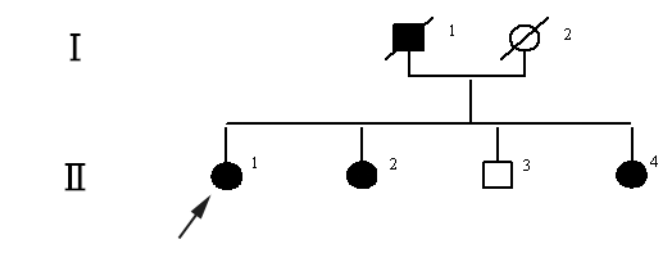

B

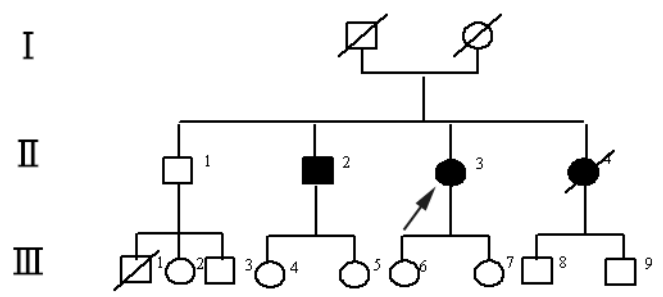

Figure 1. Pedigree of two families with the R75P mutation. Arrows indicate the proband in each family. Black-filled symbols indicate those with clinical symptoms of CADASIL.

\section{Case Family A (Fig. 1A)}

The proband of Family A was a 52-year-old woman (AII1). She was diagnosed as having depression after accouchement and received medication. At 50 years of age, she entered the hospital due to reduced activities of daily life and MRI revealed multiple cerebral infarctions. She entered hospital again for pneumonia at 51 years of age, and she became bedridden. At 52 years of age, she entered hospital for generalized convulsions and consulted the neurological department. Her father had suffered from repeated episodes of cerebral infarction and died at age 52 years. Neurological examination showed akinetic mutism, pseudobalbar palsy, and tetraplegia. EEG showed spike waves in the whole cerebrum. MRI revealed mild frontal and temporal lobe atrophy, multiple cerebral infarctions at the bilateral basal ganglia, and diffuse white matter lesions, which did not extend to the temporal pole (Fig. 2A-C).

A 49-year-old woman in Family A (AII-2) noticed shoulder stiffness and headaches beginning at age 40 years. She also experienced periods of tinnitus and vertigo. She visited a neurosurgeon because she sustained a head injury at age 49 years. Brain MRI revealed multiple infarctions at the bilateral basal ganglia and corona radiate (Fig. 2D-F). White matter high intensity was also noted on T2 and FLAIR (fluid attenuated inversion recovery) imaging, but it did not extend to the temporal pole. Neurological findings revealed no paralysis, but deep tendon reflex increased bilaterally and Babinski signs were bilaterally positive. MMSE and Hamilton scale were normal.

A 44-year-old woman in Family A (AII-4) visited a neurosurgeon with head injury, and brain atrophy was noted. She had experienced headache since age 39 years, and had experienced vertigo and dizziness since age 41 years. MRI revealed multiple infarctions in the right basal ganglia and corona radiata (Fig. 2G-I). Neurological findings revealed no abnormalities. MMSE and Hamilton's scale were normal.

\section{Case Family B (Fig. 1B)}

A 64-year-old woman in Family B (BII-3) visited a nearby hospital due to right thalamic hemorrhage in 1996. Hypertension and diabetes mellitus were noted. Her MRI revealed multiple, old cerebral infarctions (Fig. 3A-C). Repeated cerebral infarctions were seen over a 7-year period. She became bedridden and could not eat meals. Generalized tonic seizure occasionally occurred and she was transferred to hospital. Her parents and elderly brother (II-1) suffered from dementia. Her elderly brother (BII-2) and sister (BII-4) suffered from cerebral hemorrhage and infarctions, and her sister died of pneumonia at age 70 years.

Neurological examination showed akinetic mutism, pseudobalbar palsy, tetraplegia and contraction of the four extremities. Electroencephalography revealed paroxymal spikes and waves, predominantly in the left hemisphere. MRI in 2003 (Fig. 3D-F) revealed diffuse cerebral atrophy, multiple cerebral infarction at the midbrain, bilateral basal ganglia, corona radiata and diffuse white matter lesions, but these did not spread to the temporal pole. Ultrastructural analysis of the skin revealed granular osmophilic materials (GOM) within the basal lamina in the artery (Fig. 4). Ultimately, she developed pneumonia and died at 73 years of age.

\section{Mutation Analysis}

Genomic DNA was extracted from peripheral blood lymphocytes using a DNA extraction kit (Quiagen) after informed consent was obtained. All exons and the promoter region of the Notch3 gene (16) were directly sequenced using an ABI Prism 377 (Applied Biosystems, Foster City, CA). Mutations were confirmed by PCR, followed by restriction fragment length polymorphism (RFLP) assay, and were compared with 100 healthy Japanese controls.

The proband (AII-1) of Family A possessed a 302 G>C missense mutation of the Notch 3 mRNA, which resulted in an arginine to proline amino acid change at codon 75 . While this mutation was also seen in her sisters, who had abnormalities on MRI (AII-2 and 4), the mutation was not seen in her healthy brother, who had no abnormalities on MRI (AII3 ). This mutation results in the loss of an Aci 1 recognition site in exon 3. RFLP analysis revealed this mutation in the three sisters, but not in the brother. The same mutation was seen in the proband of Family B (BII-3), but not in her daughter (BIII-7), who had no abnormalities on MRI. This mutation was not seen on PCR-RFLP analysis in 100 Japanese controls (data not shown). The silent heterozygous 381 $\mathrm{C}>\mathrm{T}$ mutation, which results in the loss of the Aci 1 recognition site, was seen in both Family A (AII-1) and Family B (BII-3). This polymorphism was also seen in $32 \%$ of healthy Japanese controls (16). 


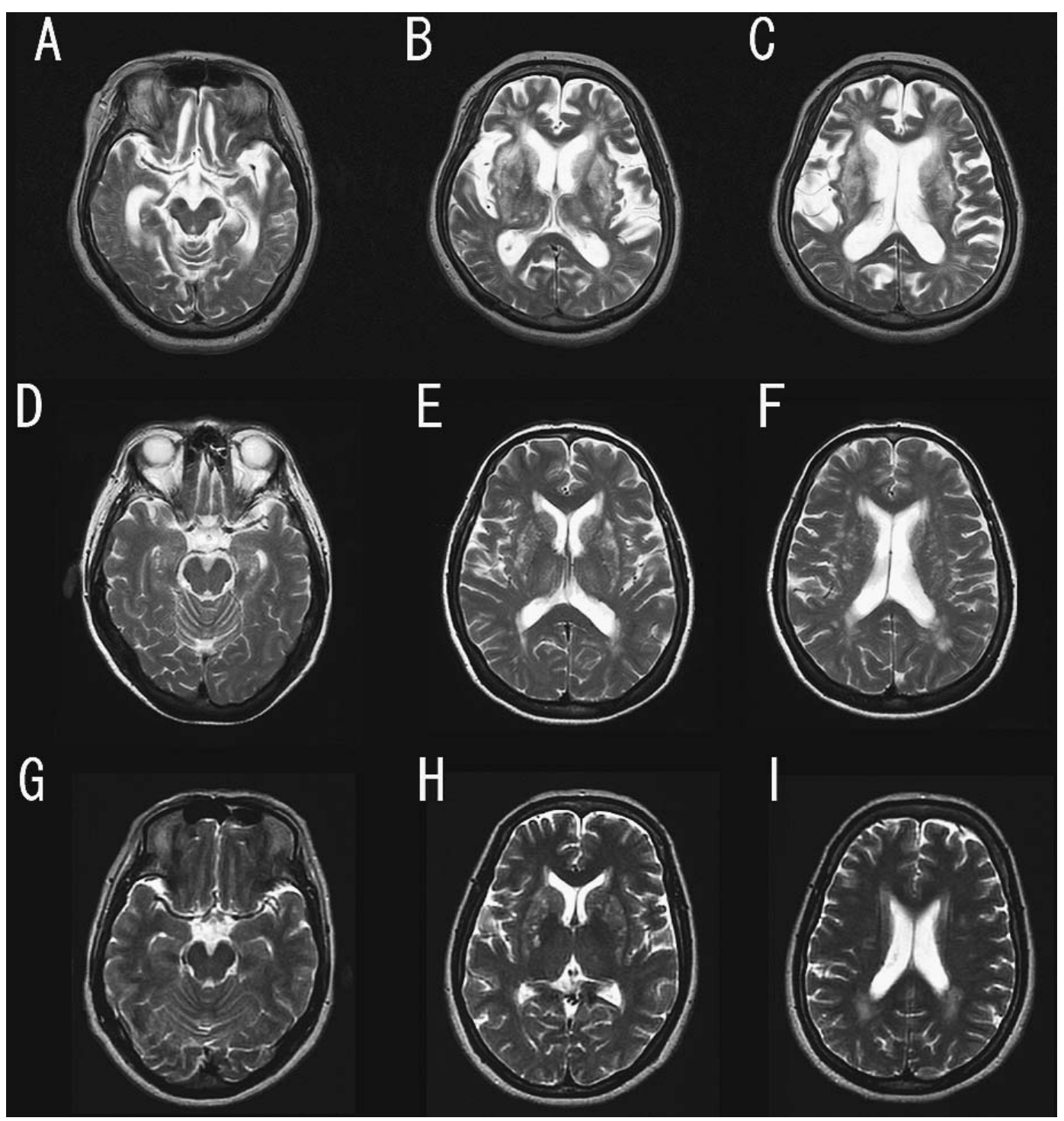

Figure 2. T2-weighted images from Family A. Multiple lacunae and white matter lesions were observed in each patient, but no lesions were detected in the temporal pole. A-C are from the proband (AII-1), D-F are from AII-2 and G-I are from AII-4.

\section{Discussion}

$\mathrm{R} 75 \mathrm{P}$ mutation is a rare mutation of Notch3 previously only reported in 4 unrelated Korean families (17). Most previous mutations in CADASIL patients were related a gain or loss of cysteine residues within an EGF-like repeat domain. An odd number of cysteine residues within an EGF-like repeat domain is thought to induce abnormal folding or dimerization, and aberrant interactions with other proteins. However, the R75P mutation does not directly involve the cysteine residues within the extracellular domain of the Notch3 as well as Italian case (7). We confirmed cosegregation of R75P mutation with development of CADASIL and found no causative mutations in other exons of the Notch3. In addition, we observed GOMs in the basal lamina of the skin vascular wall in Family B; GOMs in extracerebral arteries, including skin arterioles, are a specific hallmark of
CADASIL (18). This suggests that CADASIL is caused by the substitution of non-cysteine residues in Notch3.

The 3D structure of the first EGF-like repeat in the Notch 3 protein is predicted to be a two stranded beta-sheet followed by double (Fig. 5) (6). The six cysteines form three disulfide bonds and the double hairpin is fixed by the 5 th and 6th cysteines. Substitution of the 6th cysteine in the first EGF repeat with tryptophan at codon 76 is reported to be the cause of CADASIL (19). In the 3-dimendional studies of $\beta$-hairpin and antiparallel $\beta$-sheet structure formed by water soluble peptides, proline residues contributed to stabilize $\beta$ hairpin because proline fits well into the topography of $\beta$ turn (20). In addition, the presence of proline residues combined with disulfide bonds can lead to different conformational isoforms of both the proline and the cysteine bonds $(21,22)$. The mutation of bovine pancreatic trypsion inhibitor at the 13th proline replaced by isoleucine adjacent disulfide bridge showed conformational change and enzyme in- 


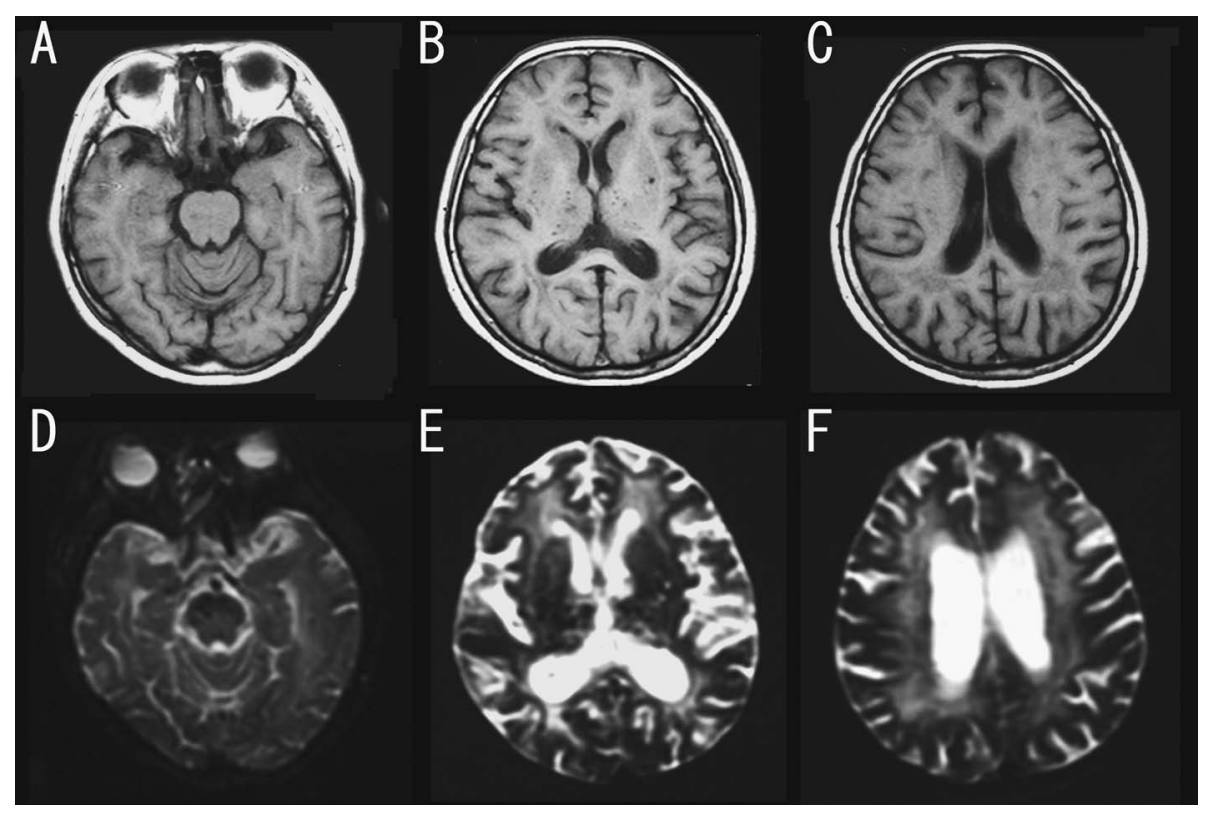

Figure 3. MR images from the proband in Family B. Multiple lacunae and white matter lesions were observed, but no lesions were detected in the temporal pole. A-C are T1-weighted images at 64 years of age, and D-F are T2-weighted images at 71 years of age.

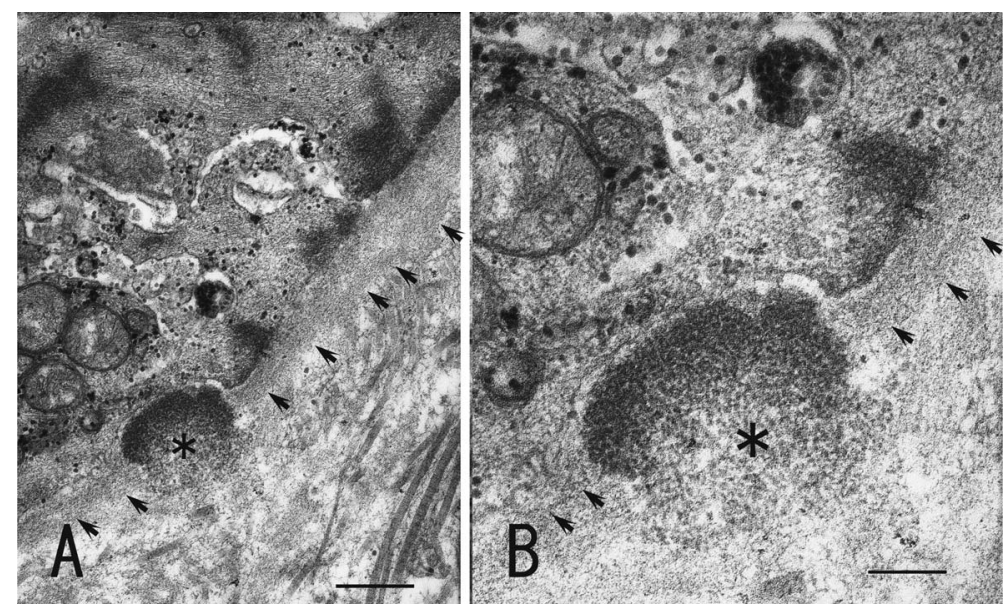

Figure 4. A. Electron micrograph of the skin vessel revealed granular osmiophilic materials (GOM) (*) within the irregular basement membrane of vascular smooth muscle cell. The arrows indicate the basement membrane. B. Higher magnification demonstrated GOM and presence of pinocytic vacuoles in the smooth muscle cell cytoplasm. The bar $=410 \mathrm{~nm}$ in $A$ and $200 \mathrm{~nm}$ in $B$.

hibitory activity (23). Dichgans et al (6) predicted that the cysteine at codon 76 would be related to fixation of the double hairpin in the EGF-like repeat of the Notch3 (Fig. 5). These previous data about the cysteine bond and proline residues support the possibility of conformational change in the EGF-like repeat by the substitution of basic arginine for the proline at codon 75 .

Interestingly, the R75P mutation has only been reported in the Korean and Japanese populations, although most mutations related to cysteine residues in CADASIL have been reported worldwide. CADASIL patients on the western coast of Finland carry the same R133C mutation, and a founder effect is suspected, as similar haplotypes are linked to the mutation in all 18 pedigrees (24). These reports suggest that a founder effect related to the R75P mutation might exist in far-eastern Asian families. On the other hand, a founder effect for other mutations in Notch3 is unlikely, as no similar hapotypes are seen in unrelated European CADASIL patients with the same missense mutations $(9,25)$. Therefore, haplotype analysis is required in order to confirm a founder effect in R75P families.

We have focused on these families for the clinical diagnosis of CADASIL because fewer white matter lesions were seen in the temporal pole on MRI. O'Sullivan et al (15) discussed the significance of temporopolar lesions as a diagnosis marker for CADASIL, and Auer et al confirmed that 


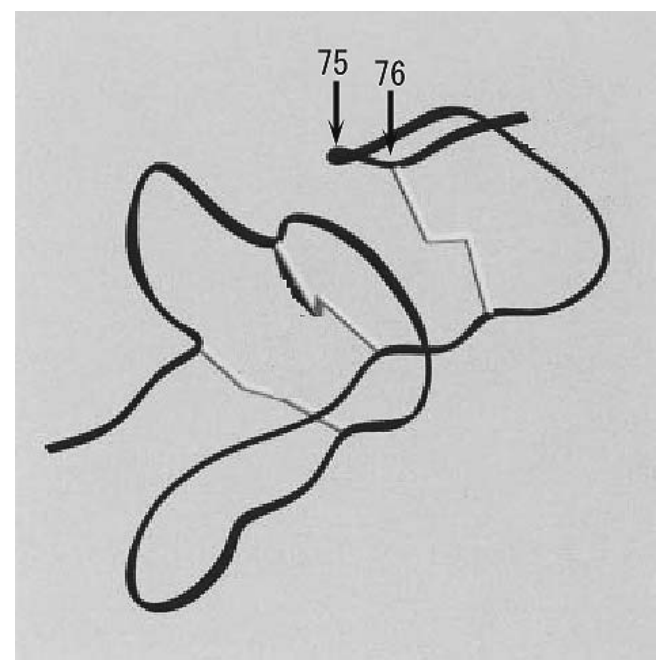

Figure 5. Three-dimensional model of the predicted main chain trace of the first Notch3 EGF-like repeat domain [modified from Dichgans et al (6)]. The Codon 75 and 76 residues are indicated by arrows.

temporopolar lesions were specific to CADASIL in Europe
(13). Tomimoto et al also reported that temporopolar lesions are diagnostic markers distinguishing CADASIL from Binswanger disease in Japanese patients, even at early stages (14). Thus, possible CADASIL patients are routinely selected for these MRI features. However, Kim et al reported less frequent white matter lesions in the anterior temporal lobe in Korean families with the R75P mutation which was true as well in the present cases (17). These cases suggested that a neurologist should pay attention to the familiar stroke patients with leukoencephalopathy in the far-eastern Asian family as a candidate for CADASIL even if MRI reveals no white matter lesion in the temporal pole. In conclusion, the R75P mutation may provide new insight into the pathogenesis of CADASIL, particularly with regard to the role of temporopolar lesions.

\section{Acknowledgement}

The authors are grateful to Dr. Kagami for the skin biopsy, Ms. Uehori for her technical support and Professor Akaji for his suggestion. This work was supported in part by a grant from the Ministry of Health, Labour and Welfare of Japan.

\section{References}

1. Tournier-Lasserve E, Joutel A, Melki J, et al. Cerebral autosomal dominant arteriopathy with subcortical infarcts and leukoencephalopathy maps to chromosome 19q12. Nat Genet 3: 256-259, 1993.

2. Joutel A, Corpechot C, Ducros A, et al. Notch3 mutations in CADASIL, a hereditary adult-onset condition causing stroke and dementia. Nature 383: 707-710, 1996.

3. Kalaria RN, Low WC, Oakley AE, et al. CADASIL and genetics of cerebral ischaemia. J Neural Transm Suppl 63 : 75-90, 2002.

4. Peters N, Opherk C, Bergmann T, Castro M, Herzog J, Dichgans M. Spectrum of mutations in biopsy-proven CADASIL: implications for diagnostic strategies. Arch Neurol 62: 1091-1094, 2005.

5. Santa Y, Uyama E, Chui de H, et al. Genetic, clinical and pathological studies of CADASIL in Japan: a partial contribution of Notch3 mutations and implications of smooth muscle cell degeneration for the pathogenesis. J Neurol Sci 212: 79-84, 2003.

6. Dichgans M, Ludwig H, Muller-Hocker J, Messerschmidt A, Gasser T. Small in-frame deletions and missense mutations in CADASIL: 3D models predict misfolding of Notch3 EGF-like repeat domains. Eur J Hum Genet 8: 280-285, 2000.

7. Mazzei R, Conforti FL, Lanza PL, et al. A novel Notch3 gene mutation not involving a cysteine residue in an Italian family with CADASIL. Neurology 63: 561-564, 2004.

8. Joutel A, Chabriat H, Vahedi K, et al. Splice site mutation causing a seven amino acid Notch3 in-frame deletion in CADASIL. Neurology 54: 1874-1875, 2000.

9. Joutel A, Vahedi K, Corpechot C, et al. Strong clustering and stereotyped nature of Notch3 mutations in CADASIL patients. Lancet 350: 1511-1515, 1997.

10. Chabriat H, Vahedi K, Iba-Zizen MT, et al. Clinical spectrum of CADASIL: a study of 7 families. Cerebral autosomal dominant arteriopathy with subcortical infarcts and leukoencephalopathy. Lancet 346: 934-939, 1995.

11. Chabriat H, Levy $C$, Taillia $H$, et al. Patterns of MRI lesions in CADASIL. Neurology 51: 452-457, 1998.

12. Coulthard A, Blank SC, Bushby K, Kalaria RN, Burn DJ. Distribution of cranial MRI abnormalities in patients with symptomatic and subclinical CADASIL. Br J Radiol 73: 256-265, 2000.

13. Auer DP, Putz B, Gossl C, Elbel G, Gasser T, Dichgans M. Dif- ferential lesion patterns in CADASIL and sporadic subcortical arteriosclerotic encephalopathy: MR imaging study with statistical parametric group comparison. Radiology 218: 443-451, 2001.

14. Tomimoto $H$, Ohtani $R$, Wakita $H$, et al. Small artery dementia in Japan: radiological differences between CADASIL, leukoaraiosis and Binswanger's disease. Dement Geriatr Cogn Disord 21: 162169, 2006.

15. O’Sullivan M, Jarosz JM, Martin RJ, Deasy N, Powell JF, Markus HS. MRI hyperintensities of the temporal lobe and external capsule in patients with CADASIL. Neurology 56: 628-634, 2001.

16. Mizuno T, Makino M, Fujiwara Y, Nakajima K. Lack of association between NOTCH3 gene polymorphism and cerebrovascular disease in Japanese patients. Ann N Y Acad Sci 977: 252-257, 2002.

17. Kim Y, Choi EJ, Choi CG, et al. Characteristics of CADASIL in Korea: a novel cysteine-sparing Notch3 mutation. Neurology 66: 1511-1516, 2006.

18. Ruchoux MM, Chabriat H, Bousser MG, Baudrimont M, TournierLasserve E. Presence of ultrastructural arterial lesions in muscle and skin vessels of patients with CADASIL. Stroke 25: 22912292, 1994.

19. Opherk C, Peters N, Herzog J, Luedtke R, Dichgans M. Longterm prognosis and causes of death in CADASIL: a retrospective study in 411 patients. Brain 127: 2533-2539, 2004.

20. Santiveri CM, Santoro J, Rico M, Jimenez MA. Factors involved in the stability of isolated beta-sheets: Turn sequence, beta-sheet twisting, and hydrophobic surface burial. Protein Sci 13: 11341147, 2004.

21. Francart C, Dauchez M, Alix AJ, Lippens G. Solution structure of R-elafin, a specific inhibitor of elastase. J Mol Biol 268: 666-677, 1997.

22. Otting G, Liepinsh E, Wuthrich K. Disulfide bond isomerization in BPTI and BPTI(G36S): an NMR study of correlated mobility in proteins. Biochemistry 32: 3571-3582, 1993.

23. Kraunsoe JA, Claridge TD, Lowe G. Inhibition of human leukocyte and porcine pancreatic elastase by homologues of bovine pancreatic trypsin inhibitor. Biochemistry 35: 9090-9096, 1996.

24. Mykkanen K, Savontaus ML, Juvonen V, et al. Detection of the 
Inter Med 47: 2067-2072, 2008 DOI: 10.2169/internalmedicine.47.1391

founder effect in Finnish CADASIL families. Eur J Hum Genet 12: 813-819, 2004.

25. Razvi SS, Davidson R, Bone I, Muir KW. The prevalence of cere- bral autosomal dominant arteriopathy with subcortical infarcts and leucoencephalopathy (CADASIL) in the west of Scotland. J Neurol Neurosurg Psychiatry 76: 739-741, 2005.

(C) 2008 The Japanese Society of Internal Medicine http://www.naika.or.jp/imindex.html 